\title{
Cysteinyl leukotriene receptor 1 gene variation and risk of asthma
}

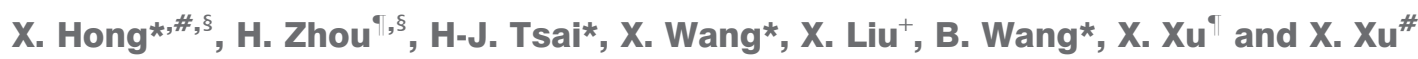

ABSTRACT: Although it has been recognised that genetics plays an important role in the development of asthma, important causal loci remain to be identified. The aim of the present study was to examine the association of known and novel candidate genes with asthma.

Two independent samples, including 170 asthmatic cases and 347 controls in the initial sample, and 202 asthmatic cases and 332 controls in the confirmation sample, were recruited from the same region of China. Functional single nucleotide polymorphisms (SNPs; $n=129)$ from 105 genes were genotyped using MassARRAY technology, and 119 SNPs were used for the subsequent analysis.

In the initial sample, three SNPs, rs320995 in the cysteinyl leukotriene receptor 1 gene, rs1047266 in the tumour necrosis factor receptor superfamily, member 10b, gene and rs40401 in the interleukin-3 gene, were associated with risk of asthma. Notably, under the recessive genetic model, subjects without the thymidine allele in SNP rs320995 had a 3.1 times higher risk of asthma, which remained significant after accounting for multiple testing. This association was replicated in the confirmation sample and validated by meta-analysis. Further, sex-specific analysis was performed, but no sex difference was found.

The present study provided coherent evidence that cysteinyl leukotriene receptor 1 gene variation is associated with risk of asthma.

KEYWORDS: Asthma susceptibility, cysteinyl leukotriene receptor 1 gene, single nucleotide polymorphism

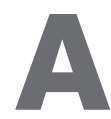
sthma, a chronic inflammatory disorder of airways, has been recognised as a serious public health problem worldwide. As estimated by the Global Initiative for Asthma in 2006, 300 million individuals of all ages and different ethnicities suffer from asthma, and the number of annual worldwide deaths due to asthma were estimated at $\sim 250,000$. Since the burden of this disease has continued increasing worldwide, better understanding of the aetiology and underlying biological mechanisms of asthma are important for the effective prevention and treatment of asthma.

It has been well established that the development of asthma is influenced by genetic and environmental factors and their interactions. Currently, $>100$ asthma susceptibility genes have been reported in at least one population [1-3]. Approximately 33 genes, which have been replicated by more than five studies, have been suggested to be underlying candidate genes of asthma [3]. However, important gaps remain in the dissection of genetic predispositions of asthma. For example, even if a genotype association is replicated, it is often associated with different phenotypes, different polymorphisms in the same gene, different alleles of the same variant or different populations [1]. Since asthma is a complex disease, genes with different biological functions and involved in various regulatory pathways may simultaneously affect the development of asthma. However, most reported genetic studies of asthma have examined only one or a few candidate genes in a single study. Only limited studies have systematically examined a large number of candidate genes in relation to asthma or asthma-related phenotypes [4-11].

The aim of the present study was to systematically examine the association of known or suspected novel candidate genes with asthma and asthma-related phenotypes in two sets of homogeneous samples recruited from the same region of China: one for an initial genetic
AFFILIATIONS

*Mary Ann and J. Milburn Smith Child Health Research Program, Children's Memorial Hospital and Children's Memorial Research Center,

\#Center for Population Genetics, Division of Epidemiology and Biostatistics, School of Public Health, University of Illinois at

Chicago, Chicago, IL,

- Program of Population Genetics, Harvard School of Public Health, Boston, MA, USA.

${ }^{+}$Institute of Biomedicine, Anhui Medical University, Hefei, China ${ }^{\S}$ Both authors contributed equally to this article.

\section{CORRESPONDENCE}

X. Xu, Center for Population Genetics, Division of Epidemiology and Biostatistics, School of Public Health M/C 923, University of Illinois at Chicago, 1603 W Taylor, Rm 978B, Chicago, IL 60612, USA Fax: 13129960064

E-mail: xipingxu@uic.edu

Received:

April 172008

Accepted after revision:

September 112008

\section{SUPPORT STATEMENT}

This study was supported by National Heart, Lung, and Blood Institute (Bethesda, MD, USA) grant R01 HL66385. The Broad Institute Center for Genotyping and Analysis (Cambridge, MA, USA) is supported by grant U54 RR020278-01 from the National Center for Research Resources (Bethesda, MD, USA).

\section{STATEMENT OF INTEREST}

None declared. 
association test, and the other for confirmation. To the present authors' knowledge, the present study is the largest asthma candidate gene study in a Chinese population.

\section{MATERIALS AND METHODS}

\section{Subject recruitment and phenotype definition}

In the present study, 170 unrelated asthmatic cases and 347 unrelated controls (the initial sample) were selected from 3,022 families. A detailed description of the study site, subject recruitment and protocols of data collection has been provided previously [12]. Briefly, the study subjects were enrolled from the eight counties of the city of Anqing (China). The following data were available for each participant: 1) information on respiratory symptoms, disease history and smoking, collected using a questionnaire; 2) forced expiratory volume in one second (FEV1) and forced vital capacity (FVC), measured using a standardised spirometric test; 3) airway methacholine challenge test results from five sequentially increasing combinations of numbers of breaths and methacholine concentration (1 breath of $1 \mathrm{mg} \cdot \mathrm{mL}^{-1}, 1$ breath of $5 \mathrm{mg} \cdot \mathrm{mL}^{-1}, 4$ breaths of $5 \mathrm{mg} \cdot \mathrm{mL}^{-1}, 1$ breath of $25 \mathrm{mg} \cdot \mathrm{mL}^{-1}$ and 4 breaths of $25 \mathrm{mg} \cdot \mathrm{mL}^{-1}$ ), with the test being terminated at the dose that produced $a \geqslant 20 \%$ fall in FEV1 from baseline (PD20), or at the final dose if PD20 was not observed; and 4) blood eosinophil (Eos) count, performed by use of a Coulter counter. The present study protocol was approved by the Human Subjects Committee (the institutional review board (IRB)) of Harvard School of Public Health (Boston, MA, USA), the institutional review board of the University of Illinois at Chicago (Chicago, IL, USA) and the Ethics Committee of Anhui Medical University (Hefei, China). Written informed consent was explained to, read and signed by each participant.

In the initial step of the present study, asthma cases were defined on the basis of: 1) self-reported physician-diagnosed asthma; 2) an observed PD20 on or before the third dose during the five-dose test; and 3) being in the top quartile of Eos distribution; The controls were defined on the basis of: 1) no history of self-reported physician-diagnosed asthma; 2) no observed PD20 during the five-dose test; and 3) being in the bottom quartile of Eos distribution. In total, 170 asthma cases and 347 controls were enrolled for genotyping.

An additional 202 unrelated asthmatic cases and 332 unrelated matched controls (the confirmation sample) were selected from the same study population in order to independently confirm the significant signal detected in the initial screening. The selection criteria for asthmatic cases and controls in the confirmation sample were almost identical to those employed for the initial sample, except that the criterion based on Eos distribution was not applied during the enrolment of the confirmation sample. It is of note that of all the cases and controls in the two data sets were unrelated.

\section{Criteria for candidate gene and single nucleotide polymorphism selection}

Asthma candidate genes were first identified from GeneCards [13]. Specifically, candidate genes were identified using the following keywords: "asthma", "bronchial responsiveness", "lung function", " airway smooth muscle", "airway allergy or inflammation", "eosinophil and allergy or inflammation", "mast cell and allergy or inflammation", "IgE", and "atopy".
A total of 989 potential asthma candidate genes were identified from the GeneCards database. Next, candidate single nucleotide polymorphisms (SNPs) were selected from among these 989 candidate genes based on the following criteria: 1) allele frequency in Asian populations (obtained from the HapMap project [14] and Affymetrix [15]); 2) BLOck SUbstitution Matrix with $80 \%$ identity (BLOSUM80) score [16], which represents the severity of amino acid change caused by a nonsynonymous $\mathrm{SNP}$, a lower score meaning more severity in amino acid change; and 3) previously reported synonymous SNPs that showed association with asthma. All SNPs with a minor allele frequency (MAF) of $<5 \%$ and all nonsynonymous SNPs with a BLOSUM80 score of $\geqslant 0$ were removed. If complete linkage disequilibrium (LD; $\mathrm{r}^{2}=1$ ) existed among two or more nonsynonymous SNPs on the same gene, only the SNP with the lowest BLOSUM80 score was included. As a result, a total of 141 SNPs from 113 genes were selected and 129 SNPs from 105 genes were successfully genotyped. Detailed information regarding each genotyped SNP is provided in table E1 of the supplementary material.

\section{Genotyping}

A venous blood sample was obtained from each study participant, and genomic DNA was extracted from blood lymphocytes using a standard salting out procedure. Genotyping of 129 SNPs was performed using Sequenom (San Diego, CA, USA) MassArray technology in the Broad Institute Center for Genotyping and Analysis (Cambridge, MA, USA). In the confirmatory stage, the SNP with the identifier rs320995 was genotyped using the TaqMan allelic discrimination method, which has been described previously [17]. A random $5 \%$ of the samples were independently repeated in order to confirm genotyping results. The concordance of these duplicated samples was $>99.5 \%$. Additionally, 50 samples that had been genotyped using Sequenom MassArray technology were randomly selected. SNP rs320995 was re-genotyped using the TaqMan allelic discrimination method in these 50 samples. The data showed that the concordant rate of these two genotyping methods was $100 \%$.

\section{Data analysis}

The primary outcome was the risk of developing asthma, and the secondary outcomes were continuous variables related to lung function, including FEV1, FVC and FEV1/FVC. For SNPs on the autosomal chromosomes, the Hardy-Weinberg equilibrium (HWE) in the control group was examined using a Chisquared test and those not in HWE were removed from further analysis. Under three different genetic models (additive, recessive and dominant), multiple logistic and linear regression models were used to estimate the effect of each SNP on asthma and lung function, separately, with adjustment for age, age squared, height, height squared, weight, current smoking status and study site. In order to account for multiple testing, a permutation of 10,000 times for 119 SNPs and four phenotypes under three genetic models were performed to obtain empirical p-values. The permutations were performed using $R$ [18].

The HWE test for the SNP on the $X$ chromosome was performed according to the method suggested by ZHENG et al. [19]. Since most loci on the $X$ chromosome are subject to $X$ chromosome inactivation, males were treated as homozygous 
females in the initial analysis, as applied previously in a genome-wide association study [20], and then the association was tested under the three genetic models. Sex-specific analysis was also performed.

Next, a meta-analysis was undertaken to evaluate the overall effect of SNP rs320995 in the cysteinyl leukotriene (Cys-LT) receptor $1 \quad\left(\right.$ CysLT $\left._{1}\right)$ gene (CYSLTR1). Published reports containing the terms "CYSLTR1 asthma", "cysteinyl leukotriene receptor 1 asthma" and "cysteinyl-leukotriene type 1 receptor asthma" were identified by PubMed searches (1966 to April 2008) by two independent investigators (X. Hong and H-J. Tsai). From the initial search, six articles were found [2126]. Three articles were excluded due to not having casecontrol study design and there being no information on sample size [23, 25, 26]. Heterogeneity was assessed using a Chisquared test, and the meta-analysis was performed using a fixed-effect model if no significant heterogeneity existed, or using a random-effect model if huge heterogeneity existed. The pooled odds ratio (OR) and $95 \%$ confidence interval (CI) were calculated as a measure of the genetic effect of rs320995 on asthma in the whole population as well as in sex-specific subgroups. It should be noted that, in sex-specific metaanalyses, the report of $\mathrm{CHOI}$ et al. [22] was excluded because it did not report sex-specific results. All of the meta-analyses were performed using $\mathrm{R}$.

\section{RESULTS}

\section{Demographic and genotyping characteristics}

The phenotypic characteristics of the 170 asthmatic cases and 347 controls in the initial sample, as well as the 332 asthmatic cases and 202 controls in the confirmation sample, are summarised in table 1 . In the initial sample, asthmatic cases were relatively younger, shorter, had a lower body mass index (BMI) and a higher percentage of males compared to controls. In the confirmation sample, asthmatic cases and controls were comparable in terms of age, sex, weight, height and BMI.

A total of 129 SNPs from 105 genes with different biological functions were genotyped in the initial sample (see table E1 of the supplementary material). Among them, 16 genes have previously been reported to be associated with asthma or asthma-related phenotypes in at least one published study. The mean genotyping call rate for the 129 SNPs was $\sim 97.5 \%$. Six SNPs with a MAF of $<0.05$ were excluded in the subsequent analyses. The mean MAF for the remaining 123 SNPs was $\sim 0.23$. Using a p-value of $<0.01$ as the cut-off for significance, four SNPs (rs3732486, rs11584340, rs2071499 and rs2274907) were not in HWE and removed from the subsequent analysis. Finally, a total of 119 SNPs from 98 genes, with a genotyping call rate of $97.9 \%$, were used to examine the association with asthma and lung function.

\section{Initial association analysis}

In the initial sample, evidence suggestive of an association with asthma was found for three of the SNPs with a pobserved of $<0.01$, including rs320995 in CYSLTR1 (OR 3.1; 95\% CI 1.85.4; pobserved $=0.00004), \mathrm{rs} 1047266$ in the tumour necrosis factor (TNF) receptor superfamily, member 10b, gene (TNFRSF10B; OR 1.9; 95\% CI 1.2-3.1; pobserved $=0.007)$ and rs40401 in the interleukin (IL)-3 gene (IL3; OR 0.5; 95\% CI 0.3-0.8; pobserved $=0.008$; table 2 ). Only the association between SNP rs320995 and asthma remained significant after accounting for multiple testing by permutation (pglobal=0.047). Interestingly, this association remained marginally significant if Bonferroni correction was applied instead of permutation.

The association between each SNP and continuous variables related to lung function are shown in table E2 of the supplementary material. SNP rs320995 tended to be associated with FEV1/FVC (pobserved=0.0004), but not with FEV1 or FVC. Evidence suggestive of association with FEV1 was also detected for four SNPs with a pobserved of $<0.01$. However, none of these associations were significant after accounting for multiple testing.

Further subgroup analyses were performed for the relationship between rs320995 and asthma in subjects aged $<25$ yrs and $\geqslant 25 \mathrm{yrs}$, and similar associations detected in the younger (OR 5.2; 95\% CI 1.7-16.5; pobserved $=0.005$ ) and older group (OR 3.0; $95 \%$ CI 1.4-6.3; pobserved $=0.004)$. There was no significant interaction between age and rs320995 by log-likelihood-ratio test.

\section{Confirmatory and pooled analyses for single nucleotide polymorphism rs320995}

The association between SNP rs320995 and asthma was successfully validated in another independent sample of 202

TABLE 1 Population characteristics of the two sample sets

\begin{tabular}{|c|c|c|c|c|}
\hline & \multicolumn{2}{|c|}{ Initial sample } & \multicolumn{2}{|c|}{ Confirmation sample } \\
\hline & Case & Control & Case & Control \\
\hline Subjects $\mathrm{n}$ & 170 & 347 & 202 & 332 \\
\hline Age yrs & $22.6 \pm 14.5^{\star \star}$ & $33.0 \pm 13.1$ & $28.0 \pm 15.2$ & $29.0 \pm 16.0$ \\
\hline Weight kg & $40.7 \pm 14.9^{\star \star}$ & $51.0 \pm 9.3$ & $44.7 \pm 15.2$ & $45.4 \pm 12.8$ \\
\hline Height $\mathrm{cm}$ & $145.1 \pm 17.4^{\star \star}$ & $157.5 \pm 9.6$ & $149.5 \pm 17.6$ & $151.9 \pm 14.3$ \\
\hline BMI $\mathbf{k g} \cdot \mathbf{m}^{-2}$ & $18.5 \pm 3.3^{\star *}$ & $20.4 \pm 2.6$ & $19.2 \pm 3.4$ & $19.2 \pm 3.0$ \\
\hline \multicolumn{5}{|l|}{$\begin{array}{l}\text { Asthma-related } \\
\text { phenotype }\end{array}$} \\
\hline FEV 1 L & $2.1 \pm 0.8^{\star \star}$ & $3.1 \pm 0.7$ & $2.3 \pm 0.8^{\star \star}$ & $3.0 \pm 0.9$ \\
\hline FVC L & $3.1 \pm 1.2^{* *}$ & $3.7 \pm 0.9$ & $3.3 \pm 1.2^{*}$ & $3.5 \pm 1.1$ \\
\hline $\mathrm{FEV}_{1} / \mathrm{FVC}$ & $0.7 \pm 0.1^{* *}$ & $0.8 \pm 0.1$ & $0.7 \pm 0.1^{\star \star}$ & $0.9 \pm 0.1$ \\
\hline $\log _{10}$ Eos cells $\cdot \mathrm{mm}^{-3}$ & $2.7 \pm 0.2^{\star \star}$ & $1.4 \pm 0.1$ & $2.0 \pm 0.5^{\star \star}$ & $1.9 \pm 0.3$ \\
\hline Male & $87(51.2)^{*}$ & $145(41.8)$ & $119(58.9)$ & $196(59.0)$ \\
\hline Children $^{\#}$ & $90(52.9)^{\star \star}$ & $52(15.0)$ & $69(34.2)$ & $123(37.1)$ \\
\hline Current smoker & $17(21.3)$ & $76(25.8)$ & $54(26.7)$ & $85(25.6)$ \\
\hline \multicolumn{5}{|l|}{ Study sites } \\
\hline Huanning county & $42(12.1)$ & $9(5.3)$ & $49(14.8)$ & 37 (18.3) \\
\hline Qianshan county & $18(5.2)$ & $24(14.1)$ & $53(16.0)$ & $18(8.9)$ \\
\hline Susong county & $16(4.6)$ & $20(11.8)$ & $11(3.3)$ & $12(5.9)$ \\
\hline Tongchen county & $9(2.6)$ & $12(7.1)$ & $5(1.5)$ & $10(4.9)$ \\
\hline Taihu county & $31(8.9)$ & $23(13.5)$ & $9(2.7)$ & $24(11.9)$ \\
\hline Wangjiang county & $174(50.1)$ & $34(20.0)$ & $82(24.7)$ & $50(24.8)$ \\
\hline Yuexi county & $8(2.3)$ & $7(4.1)$ & $48(14.5)$ & $10(5.0)$ \\
\hline Zhongyang county & $49(14.1)^{\star \star}$ & $41(24.2)$ & $75(22.6) * \star$ & $41(20.3)$ \\
\hline
\end{tabular}

Data are presented as mean \pm SD for continuous variables and $n(\%)$ for categorical variables. BMI: body mass index; FEV1: forced expiratory volume in

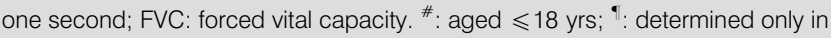
adults. ${ }^{*}: p<0.05 ;{ }^{* *}: p<0.01$ versus controls (unpaired t-test for continuous variable and Chi-squared test for categorical variables) 
TABLE 2 Association of single nucleotide polymorphisms (SNPS) with the risk of developing asthma in the initial screening\#

\begin{tabular}{|c|c|c|c|c|c|c|c|c|}
\hline Gene & SNP ID & Polymorphism & MAF" & \multicolumn{2}{|c|}{ Genotypic distribution $^{+}$} & \multicolumn{3}{|c|}{ Genotypic analysis } \\
\hline TNFRSF10B & rs1047266 & Ala67Val & 26.9 & $69 / 77 / 21$ & $186 / 128 / 28$ & Dominant & $1.9(1.2-3.1)$ & 0.007 \\
\hline IL3 & rs40401 & Ser27Pro & 49.7 & $46 / 94 / 30$ & $93 / 163 / 91$ & Recessive & $0.5(0.3-0.8)$ & 0.008 \\
\hline RNASE3 & rs2073342 & Thr124Arg & 27.5 & $75 / 81 / 14$ & $182 / 136 / 27$ & Dominant & $1.8(1.1-2.9)$ & 0.011 \\
\hline SERPINB2 & rs6104 & Ser413Cys & 44.0 & $38 / 94 / 37$ & $100 / 183 / 59$ & Additive & $1.5(1.0-2.1)$ & 0.026 \\
\hline TPO & rs1126799 & Val847Ala & 37.1 & $70 / 61 / 29$ & $130 / 163 / 43$ & Dominant & $0.6(0.4-1.0)$ & 0.042 \\
\hline MMP8 & rs3765620 & Ile32Thr & 39.1 & $75 / 71 / 21$ & $129 / 161 / 54$ & Recessive & $0.5(0.2-1.0)$ & 0.040 \\
\hline
\end{tabular}

ID: identifier; MAF: minor allele frequency; OR: odds ratio; CI: confidence interval; CYSLTR1: cysteinyl leukotriene receptor 1 gene; TNFRSF1OB: tumour necrosis factor receptor superfamily, member 10b gene; IL3: interleukin-3 gene; RNASE3: ribonuclease, RNase A family, 3 gene; CSF2: colony-stimulating factor 2 (granulocyte macrophage) gene; SERPINB2: serpin peptidase inhibitor, clade B, member 2 gene; TPO: thyroid peroxidase gene; MMP8: matrix metalloprotease-8 gene; Phe309Phe: substitution of phenylalanine 309 with phenylalanine; Ala: alanine; Val: valine; Ser: serine; Pro: proline; Thr: threonine; Arg: arginine; lle: isoleucine; Cys: cysteine. \#: pobserved $<0.05$; ": in control sample; ${ }^{+}$: shown as homozygotes for major allele/heterozygotes/homozygotes for minor allele; ${ }^{\text {s: }}$ most proximate model (of recessive, dominant and additive); ${ }^{\text {: }}$ adjusted for age, age squared, height, height squared, weight, current smoking, sex and study site; ${ }^{\# \#: ~ p-v a l u e ~ n o t ~ a c c o u n t i n g ~ f o r ~ m u l t i p l e ~}$ testing; " : pglobal $=0.047$ after accounting for multiple testing by permutation.

asthmatic cases and 332 controls (OR 1.6; 95\% CI 1.0-2.4; $\mathrm{p}=0.032$ ), as well as in the pooled sample (OR 2.0; 95\% CI 1.42.7; $\mathrm{p}=0.00002$; table 3).

Next, sex-stratified analyses were performed separately in each independent sample and in the pooled sample. The present results suggested that males without a T allele in SNP rs320995 had a significantly higher risk of asthma in the initial sample (OR 3.2; 95\% CI 1.5-7.1; p=0.004), confirmation sample (OR $1.7 ; 95 \%$ CI 1.0-2.9; $\mathrm{p}=0.045)$ and pooled sample (OR 2.1; $95 \%$ CI 1.4-3.1; $\mathrm{p}=0.0003$; table 3). Likewise, under the genetic recessive model, females without a T allele in SNP rs320995 showed a significantly higher risk of asthma in the initial sample (OR 3.3; 95\% CI 1.5-7.3; $\mathrm{p}=0.003$ ) and pooled sample
(OR 2.0; 95\% CI 1.1-3.4; $\mathrm{p}=0.014$ ). The effect of SNP rs320995 on risk of asthma was comparable in males and females.

Finally, the association of SNP rs320995 with lung function in the confirmation and pooled samples was examined. It was found that, in males, the relationship between FEV1/FVC and SNP rs320995 in the confirmation sample and pooled sample showed a similar trend to that detected in the initial sample (see table E3 of the supplementary material).

Meta-analysis of single nucleotide polymorphism rs320995 Meta-analysis was performed to estimate the genetic effect of rs320995 on asthma using the present data and data available from previous published reports, for which mild heterogeneity

TABLE 3 Association of rs320995 with risk of developing asthma in a Chinese population

\begin{tabular}{|c|c|c|c|c|c|c|c|c|c|}
\hline \multirow[t]{2}{*}{ Allele } & \multicolumn{3}{|c|}{ Initial sample } & \multicolumn{3}{|c|}{ Confirmation sample } & \multicolumn{3}{|c|}{ Pooled sample } \\
\hline & Case & Control & $\mathrm{OR}^{\#}$ & Case & Control & $\mathrm{OR}^{\#}$ & Case & Control & $\mathrm{OR}^{\#}$ \\
\hline Subjects n & 170 & 343 & & 202 & 332 & & 372 & 675 & \\
\hline \multicolumn{10}{|l|}{ Total } \\
\hline $\mathrm{T}$ & 67.6 & 82.5 & 1.0 & 69.8 & 77.4 & 1.0 & 69.5 & 80.9 & 1.0 \\
\hline$T$ & 56.3 & 74.7 & 1.0 & 60.5 & 70.4 & 1.0 & 58.7 & 72.2 & 1.0 \\
\hline No $T^{\circ}$ & 43.7 & 25.4 & $3.2(1.4-7.1)^{\star \star}$ & 39.5 & 29.6 & $1.7(1.0-2.9)^{\star}$ & 41.3 & 27.8 & $2.1(1.4-3.1)^{\star \star \star}$ \\
\hline \multicolumn{10}{|l|}{ Female } \\
\hline $\mathrm{T}$ & 79.5 & 88.1 & 1.0 & 83.1 & 87.5 & 1.0 & 81.3 & 87.8 & 1.0 \\
\hline No $T^{*}$ & 20.5 & 11.9 & $3.3(1.5-7.3)^{\star \star}$ & 16.9 & 12.5 & $1.4(0.6-3.3)$ & 18.7 & 12.2 & $2.0(1.1-3.4)^{\star}$ \\
\hline
\end{tabular}

Data are presented as percentages unless otherwise indicated. OR; odds ratio; T: thymidine. \#: adjusted for age, age squared, height, height squared, weight, current smoking, sex (only for the analysis in the total population) and study site; ": males with a cytosine (C) allele or females of CC genotype. *: $p<0.05 ;{ }^{* \star}: \mathrm{p}<0.01$; $* * *: p<0.001 ;+: p<0.0001$. 


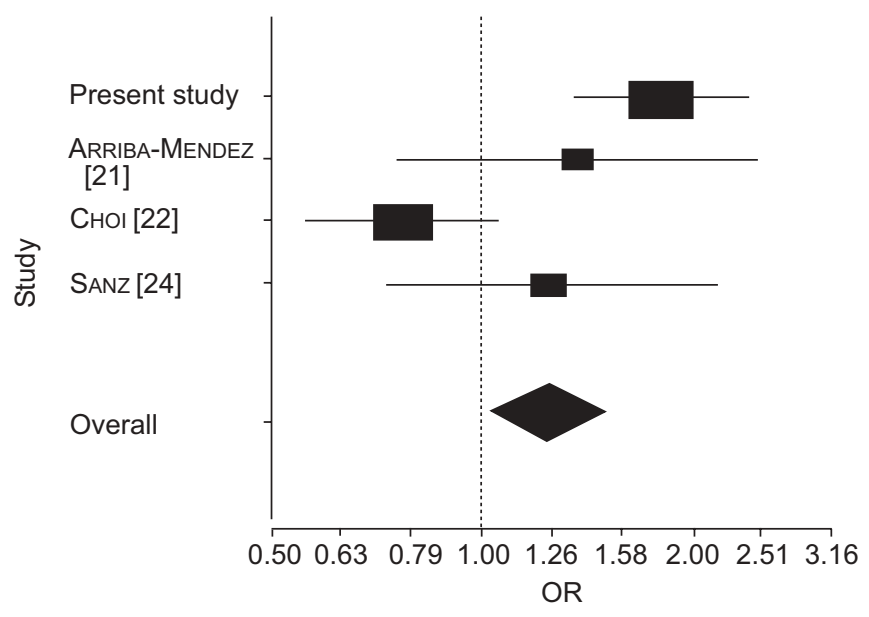

FIGURE 1. Pooled analysis of effect of single nucleotide polymorphism rs320995 on asthma. Data are presented as odds ratio (OR; ( (size reflects weighting)), using a fixed effect model ( $\cdots$... line of no effect), and $95 \%$ confidence interval (horizontal bars). The centre of the diamond indicates the combined mean effect of the studies included in meta-analysis and its extremities the $95 \%$ confidence interval.

was observed $(\mathrm{p}=0.03)$. A significant association was found between rs320995 and asthma using a fixed-effect model (OR $1.3 ; 95 \%$ CI 1.1-1.5; fig. 1) and marginally significant association using a random-effect model (OR 1.2; 95\% CI 0.8-1.9). On sex-specific analysis, heterogeneity was not observed and a significant association was detected between rs320995 and asthma in both males (OR 2.0; 95\% CI 1.4-2.8) and females (OR $1.4 ; 95 \%$ CI 1.0-2.1) using a fixed-effect model.

\section{DISCUSSION}

Taken together, the present study has contributed to the genetics of asthma in the following aspects. First, the coding SNPs of 98 asthma candidate genes were systematically examined in relation to asthma and lung function. To the present authors' knowledge, this is the largest-scale asthma candidate gene study in a homogeneous Chinese population.

Secondly, a significant association between SNP rs320995 in CYSLTR1 and the risk of asthma was validated in two independent Chinese samples. Although the associations between CYSLTR1 variations and asthma or asthma-related phenotypes have previously been investigated in Caucasian $[21,23,24]$ and Asian populations [22, 25-28], the results were inconsistent. In detail, ARRIBA-MENDEZ et al. [21] reported that the CYSLTR1 thymidine to cytosine substitution at nucleotide 927 (rs320995) variation could predispose male children to asthma and atopic dermatitis in a Caucasian population [21]. This positive association was confirmed by SANZ et al. [24] in an adult Caucasian population and by KIM and co-workers [27, 28] in an adult Korean population, but KIM and co-workers [27, 28] presented a positive relationship between the promoter variations and the risk of aspirin-intolerant asthma. Inconsistent with these findings, two studies in a Japanese population found no association of the promoter variations or rs320995 in CYSLTR1 with asthma [25, 26]. The study in a Caucasian population found that rs320995 was associated with female atopic severity but not asthma [23]. These inconsistent results may be partly caused by limited statistical power in some previous reports of relatively small sample size $(\mathrm{n}<400)$. Another possible explanation for the inconsistency is the genetic heterogeneity of asthma and ethnicity/geometric difference, which suggests that the association between CYSLTR1 variations and asthma should be replicated in different populations. The results of the current meta-analysis using the present data and data from three previous reports $[21,22,24]$ further support the genetic effect of SNP rs320995 on asthma.

The association between CYSLTR1 variation and risk of asthma is biologically plausible. CYSLTR1 encodes the G-proteincoupled receptor CysLT 1 . Cys-LTs are biologically active lipid mediators, which can promote human airway smooth muscle contraction, proliferation and migration [29]. Cys-LTs have also been reported to be involved in the generation of type-2 T-helper cell cytokines and expression of some mediators involved in the process of airway inflammation and remodelling, such as TNF- $\alpha$, endothelin 1 [30], reactive oxygen intermediate [31], histamine receptor [32] and IL-11 [33]. These biological actions of Cys-LTs occur via binding to CysLT receptors, CysLT 1 and CysLT 2 [34]. Activation of CysLT may result in proliferation and contraction of smooth muscle, oedema and Eos migration to the lungs, and thus play an important role in the development of asthma. In the present study, evidence was found suggestive that SNP rs320995 may affect asthma and also FEV1/FVC, but not FEV1 or FVC. Given the multiple functions of CYSLTR1, the specific pathways by which SNP rs320995 affects the risk of asthma should be further investigated.

Although SNP rs320995 does not cause any change in amino acid sequence, it remains likely that the nucleotide substitution at SNP rs320995 might affect the efficiency of CYSLTR1 mRNA processing and/or stability. Alternatively, SNP rs320995 may be in strong or complete LD with some important functional SNPs in CYSLTR1, which are asthma causal. Therefore, the available SNPs in CYSLTR1 were explored in a Chinese population from the HapMap project and it was found that SNP rs320995 was in strong LD with SNP rs3201029 in the promoter region $\left(\mathrm{r}^{2}=0.87\right)$. In addition, published data indicated that SNP rs320995 was in complete LD with SNPs rs3201029, rs2637204 and rs2806489 in the promoter regions [25]. These polymorphisms have been reported to significantly change promoter activity and increase the risk in the development of aspirin-intolerant asthma [25, 27, 28]. As such, SNP rs320995 may be a surrogate marker for a causal SNP in the promoter region of CYSLTR1. In the future, it might be more informative to map the causal SNP(s) in CYSLTR1 by genotyping the complete SNP set covering the whole gene.

In addition to SNP rs320995, the present findings also suggested that SNP rs40401 in IL3 should be of particular interest for further investigation. Specifically, the present study validated the protective effect of the proline 27 allele in SNP rs40401 in IL3 on asthma, which has been reported previously in a Korean population [35]. Similar associations were detected between this SNP and lung function, including FVC and FEV1/FVC. Although the results did not remain significant after correcting for multiple testing, this is probably due to a modest genetic effect and/or the relatively small sample size. 
The present study has several strengths. First, asthmatic cases were defined carefully based on physician diagnosis plus airway responsiveness to methacholine challenge. This combined definition is more reliable than physician diagnosis alone since: 1) clinical definition of asthma relies on the clinical experience of physicians and thus lacks standardisation; and 2) airway responsiveness to methacholine is a reproducible and robust method associated with the diagnosis of asthma [36]. Secondly, all of the participating subjects were recruited from a rural area and were homogeneous with respect to ethnicity, lifestyle and occupation, as well as social and cultural norms, which would minimise potential confounding effects and decrease the degree of false-positive results. Thirdly, the association between SNP rs320995 in CYLSTR1 and asthma was identified and confirmed in two independent samples. Since false-positive association has been a critical concern in candidate-gene association studies, the approach herein would ensure that the observed association was less likely to be a false-positive result.

However, there are several limitations that should be considered. In the initial sample, the controls were older than the cases. This was intentional and done to ensure that controls would not develop asthma. In order to minimise the potential confounding effects caused by age, height and weight, these three variables were included as covariates in all of the analyses, and the observed association was further confirmed in an independent set of cases and controls with matched characteristics in terms of age, height and weight. The present authors acknowledge that such study design may be problematic in terms of detection of age-related associations. However, in the present study, similar associations between rs320995 and asthma were detected in both age groups and no significant interaction was found between age and rs320995, which suggests that age may have a minimal effect on the association between rs320995 and asthma. Another limitation is that the cases and controls in the initial sample were selected based on an additional criterion, which was not applied in the confirmation sample, i.e. the cases/ controls in the initial sample had an extremely high/low Eos level. Since no association could be observed between SNP rs320995 and Eos level in the confirmation sample, and the association between SNP rs320995 and asthma remained significant with additional adjustment of logarithmically transformed Eos density in the pooled sample, it was possible to be confident that such sample selection would not lead to a bias in the present findings. Finally, total immunoglobulin E data were not available for the study, which limited ability to explore the association of rs320995 with atopy.

In conclusion, the present study provides strong evidence that single nucleotide polymorphism rs320995 in the cysteinyl leukotriene receptor 1 gene is associated with asthma in a Chinese population, and that this effect is comparable in males and females. Further investigation into the biological functions of the cysteinyl leukotriene receptor 1 gene in relation to asthma and its related phenotypes may provide new clues for asthma therapeutic and preventive strategies.

\section{ACKNOWLEDGEMENTS}

The authors acknowledge the assistance and cooperation of the faculty and staff of the Anhui Institute of Biomedicine (Anhui Medical University, Hefei, China) and thank all study participants for their support.

\section{REFERENCES}

1 Ober C, Hoffjan S. Asthma genetics 2006: the long and winding road to gene discovery. Genes Immun 2006; 7: 95-100.

2 Bosse Y, Hudson TJ. Toward a comprehensive set of asthma susceptibility genes. Annu Rev Med 2007; 58: 171-184.

3 Vercelli D. Discovering susceptibility genes for asthma and allergy. Nat Rev Immunol 2008; 8: 169-182.

4 Hoffjan S, Ostrovnaja I, Nicolae D, et al. Genetic variation in immunoregulatory pathways and atopic phenotypes in infancy. J Allergy Clin Immunol 2004; 113: 511-518.

5 Schubert K, von Bonnsdorf $\mathrm{H}$, Burke M, et al. A comprehensive candidate gene study on bronchial asthma and juvenile idiopathic arthritis. Dis Markers 2006; 22: 127-132.

6 Litonjua AA, Lasky-Su JA, Schneiter $\mathrm{K}$, et al. ARG1 is a novel bronchodilator response gene: screening and replication in four asthma cohorts. Am J Respir Crit Care Med 2008; 178: 688-694.

7 Sharma M, Batra J, Mabalirajan U, et al. A genetic variation in inositol polyphosphate 4 phosphatase A enhances susceptibility to asthma. Am J Respir Crit Care Med 2008; 177: 712-719.

8 Kurz T, Hoffjan S, Hayes MG, et al. Fine mapping and positional candidate studies on chromosome 5p13 identify multiple asthma susceptibility loci. J Allergy Clin Immunol 2006; 118: 396-402.

9 Ryu HJ, Jung HY, Park JS, et al. Gene-based single nucleotide polymorphisms and linkage disequilibrium patterns of 29 asthma candidate genes in the chromosome 5q31-33 region in Koreans. Int Arch Allergy Immunol 2006; 139: 209-216.

10 Jinnai N, Sakagami T, Sekigawa T, et al. Polymorphisms in the prostaglandin $E_{2}$ receptor subtype 2 gene confer susceptibility to aspirin-intolerant asthma: a candidate gene approach. Hum Mol Genet 2004; 13: 3203-3217.

11 Hakonarson H, Bjornsdottir US, Ostermann E, et al. Allelic frequencies and patterns of single-nucleotide polymorphisms in candidate genes for asthma and atopy in Iceland Am J Respir Crit Care Med 2001; 164: 2036-2044.

$12 \mathrm{Xu}$ X, Niu T, Chen C, et al. Association of airway responsiveness with asthma and persistent wheeze in a Chinese population. Chest 2001; 119: 691-700.

13 Weizmann Institute of Science. GeneCards. www.genecards. org/index.shtml Date last updated: 21 September 2008. Date last accessed: 18 November 2008.

14 International HapMap Project. www.hapmap.org/cgi-perl/ gbrowse/ Date last updated: March 2008. Date last accessed: 3 June 2008.

15 Affymetrix. Assays \& Reagents. www.affymetrix.com/ products/reagents/specific/application_specific.affx Date last updated: 23 June 2006. Date last accessed: 25 September 2006.

16 Henikoff $S$, Henikoff JG. Amino acid substitution matrices from protein blocks. Proc Natl Acad Sci U S A 1992; 89: 10915-10919.

17 Hong X, Hsu YH, Terwedow H, et al. CYP19A1 polymorphisms are associated with bone mineral density in Chinese men. Hum Genet 2007; 121: 491-500.

18 R Project. The R Project for Statistical Computing. http:/ / www.r-project.org/ Date last updated: 22 September 2008. Date last accessed: 10 October 2008. 
19 Zheng G, Joo J, Zhang C, Geller NL. Testing association for markers on the X chromosome. Genet Epidemiol 2007; 31: 834-843.

20 Genome-wide association study of 14,000 cases of seven common diseases and 3,000 shared controls. Nature 2007; 447: 661-678.

21 Arriba-Mendez S, Sanz C, Isidoro-Garcia M, et al. 927T $>$ C polymorphism of the cysteinyl-leukotriene type-1 receptor (CYSLTR1) gene in children with asthma and atopic dermatitis. Pediatr Allergy Immunol 2006; 17: 323-328.

22 Choi JH, Park HS, Oh HB, et al. Leukotriene-related gene polymorphisms in ASA-intolerant asthma: an association with a haplotype of 5-lipoxygenase. Hum Genet 2004; 114: 337-344.

23 Hao L, Sayers I, Cakebread JA, et al. The cysteinylleukotriene type 1 receptor polymorphism $927 \mathrm{~T} / \mathrm{C}$ is associated with atopy severity but not with asthma. Clin Exp Allergy 2006; 36: 735-741.

24 Sanz C, Isidro-Garcia M, Davila I, Moreno E, Laffond E, Lorente F. Analysis of 927T >C CYSLTRI and $-444 A>C$ LTC4S polymorphisms in patients with asthma. J Investig Allergol Clin Immunol 2006; 16: 331-7.

25 Zhang J, Migita O, Koga M, Shibasaki M, Arinami T, Noguchi E. Determination of structure and transcriptional regulation of CYSLTR1 and an association study with asthma and rhinitis. Pediatr Allergy Immunol 2006; 17: 242-249.

26 Unoki M, Furuta S, Onouchi Y, et al. Association studies of 33 single nucleotide polymorphisms (SNPs) in 29 candidate genes for bronchial asthma: positive association a T924C polymorphism in the thromboxane A2 receptor gene. Hum Genet 2000; 106: 440-446.

27 Kim SH, Yang EM, Park HJ, Ye YM, Lee HY, Park HS. Differential contribution of the CysLTR1 gene in patients with aspirin hypersensitivity. J Clin Immunol 2007; 27: 613-619.

$28 \mathrm{Kim}$ SH, Oh JM, Kim YS, et al. Cysteinyl leukotriene receptor 1 promoter polymorphism is associated with aspirin-intolerant asthma in males. Clin Exp Allergy 2006; 36: 433-439.

29 Parameswaran K, Cox G, Radford K, Janssen LJ, Sehmi R, O'Byrne PM. Cysteinyl leukotrienes promote human airway smooth muscle migration. Am J Respir Crit Care Med 2002; 166: 738-742.

30 Patrignani P, Modica R, Bertolero F, Patrono C. Differential effects of leukotriene $\mathrm{C} 4$ on endothelin-1 and prostacyclin release by cultured vascular cells. Pharmacol Res 1993; 27: 281-285.

31 Bisgaard $\mathrm{H}$, Loland L, Oj JA. NO in exhaled air of asthmatic children is reduced by the leukotriene receptor antagonist montelukast. Am J Respir Crit Care Med 1999; 160: 1227-1231.

32 Pynaert G, Grooten J, van Deventer SJ, Peppelenbosch MP. Cysteinyl leukotrienes mediate histamine hypersensitivity ex vivo by increasing histamine receptor numbers. Mol Med 1999; 5: 685-692.

33 Lee KS, Kim SR, Park HS, et al. Cyseinyl leukotriene upregulates IL-11 expression in allergic airway disease of mice. J Allergy Clin Immunol 2007; 119: 141-149.

34 Hui Y, Funk CD. Cysteinyl leukotriene receptors. Biochem Pharmacol 2002; 64: 1549-1557.

35 Park BL, Kim LH, Choi YH, et al. Interleukin 3 (IL3) polymorphisms associated with decreased risk of asthma and atopy. J Hum Genet 2004; 49: 517-527.

36 James A, Ryan G. Testing airway responsiveness using inhaled methacholine or histamine. Respirology 1997; 2: 97-105. 